\title{
THERMAL ENTRANCE EFFECTS IN A THERMOACOUSTIC STACKED SCREEN REGENERATOR
}

\author{
Simon Bühler ${ }^{1}$, Douglas Wilcox ${ }^{2}$, Joris P. Oosterhuis ${ }^{1}$, Theo H. Van der Meer ${ }^{1^{*}}$ \\ ${ }^{1}$ Thermal Engineering, University of Twente, P.O. Box 217, Enschede, 7500AE, The Netherlands \\ ${ }^{2}$ Chart Inc.-Qdrive, 302 10th Street, Troy, NY 12180, United States
}

\begin{abstract}
Thermoacoustic cryocoolers are of raising interest because they are cost effective and reliable. The underlying heat pumping process occurs in the regenerator, where a sound wave interacts with a solid matrix material. Stacked screens are frequently used to build regenerators for thermoacoustic applications because of their favorable thermal properties and vast mesh sizes. One dimensional codes are commonly used for estimating the performance of thermoacoustic cryocoolers. While these codes are a useful tool in thermoacoustic device design, they do not incorporate entrance effects at the extremities of the regenerator or the resulting convective effects. In this paper these effects are investigated by means of a full Navier-Stokes solution of the flow and heat transfer in a geometrical reduced model of a regenerator. It is shown that convective effects play a role at low pressure amplitudes. A convection driven heat pumping process occurring at the extremities of the regenerator is described. Furthermore, a geometrical study is conducted to estimate the optimal opening of the stacked screen for ideal heat transfer.
\end{abstract}

KEY WORDS: Numerical simulation and super-computing, Porous media, Thermoacoustic, Stacked screen Regenerator

\section{INTRODUCTION}

Thermoacoustic cryocoolers are of raising interest as they do not have any moving parts within the cold section, making them cost effective and reliable cryocoolers. The underlying thermodynamic cycle is a Stirling cycle. However, unlike a Stirling cryocooler which makes use of a mechanical piston to perform the displacement and compression of the working fluid, in thermoacoustic based devices the cycle is executed by an acoustic wave thermally interacting with the solid material of the regenerator. The acoustic power is used to pump heat from the cold heat exchanger to the ambient heat exchanger, which are placed at the ends of the regenerator.

Due to the desired thermoacoustic effect, heat pumping occurs within one thermal penetration depth of the solid, ideally resulting in the local gas temperature following the regenerator temperature. Gas parcels further than a thermal penetration depth away from the solid undergo adiabatic wave propagation as the parcel is too far from the solid to intimately interact with it. In order to achieve a high power density the "dead" gas volume not interacting with the solid should be reduced as much as possible. For good thermal contact narrow pores are needed. However, if the pores are too small viscous losses become dominant and can lead to inefficiencies. Hence, both the thermal and viscous effects have to be balanced in the thermoacoustic regenerator.

Heat conduction along the temperature gradient in the wave propagation direction is an unwanted effect and can be seen as a loss. One way of minimizing this effect is to use stacked screens as they have only tiny contact points in the direction of the temperature gradient, while the vast mesh sizes readily available allow 
easy adjustment of the open area in the wave propagation direction for optimal balancing of the thermal and viscous effects [1].

For the design of thermoacoustic cryocoolers, one dimensional codes such as DeltaEC [2] and Sage [3] are used. The underlying thermoacoustic equations are derived from the Navier-Stokes equations by linearizing them and assuming a time harmonic behavior of the principal fluid quantities. These codes give a rough estimate of the total cryocooler performance, but they are not adequate to gain a deeper understanding of the heat transfer occurring within the regenerator as they neglect for example convective effects.

For this reason this paper investigates the heat transfer occurring within the stacked screens that make up a regenerator using computational fluid dynamics (CFD). It shall especially be focused on the entrance effects at both ends of the regenerator as they are not accurately predicted by the one dimensional acoustic codes, but have a large impact on the devices efficiency.

The presented model consists of a pair of cylinders in an inline arrangement subject to an acoustically oscillating flow. Most of the research described in literature on oscillating flows around pairs of cylinders focuses on the hydrodynamic forces and vortex generation. Experimental investigations for one and two cylinders have been carried out for example by Williamson [4]. An et al. [5] used finite element simulations to determine the force acting on the two cylinders in tandem and to reveal the flow field. Anagnostopoulos et al. [6] did the same for four cylinders in a square arrangement.

Heat transfer on one cylinder in pure oscillating flow was also investigated with simulations and experiments. Mozurkewich [7] investigated the heat transfer around a single cylinder in a standing wave experimentally. Gopinath et al. [8] carried out experiments for low amplitude oscillatory flow, for which the displacement amplitude is small compared to the cylinder diameter. Iwai et al. [9] used numerical simulations and experiments to investigate the heat transfer from one cylinder for displacement amplitudes much higher than the cylinder diameter.

In this paper the heat transfer to and from the screen mesh is investigated, by looking at two cylinders in an inline arrangement. The displacement amplitude is about ten times the cylinder diameter and the pressure and velocity oscillation are in phase. Flow effects such as convective heat transport, which are not resolved by one dimensional acoustic codes, are described. The amount of heat pumped is compared with the acoustic power dissipated in the stacked screen regenerator model and the optimal mesh size of the screen is investigated.

\section{METHOD}

The entrance effects at the end of a regenerator have a large influence on the efficiency of a thermoacoustic cryocooler. These effects are investigated using CFD simulations. The reduced numerical model and the numerical parameters are presented in this section.

2.1 CFD model As the investigation of a full 3D model of the regenerator would have a high computational cost, we reduce the regenerator here to only two screens to investigate the entrance effects at the regenerator ends. Neglecting gravity effects and the effects of the duct where the regenerator is housed, and by implementing periodic boundary conditions, the mesh can be reduced to one repetitive unit. In a first approach shown below, this cell is further reduced to a two dimensional array of cylinders, representing the individual wires of the screen. The reference model investigated in this paper is shown in Figure 1.

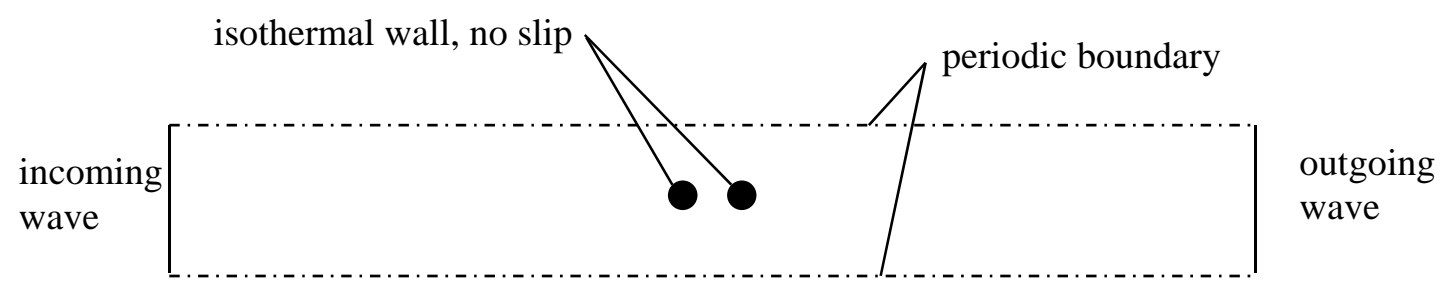

Fig. 1 Reduced model of the stacked screen with two screens. 
The simulations are carried out with the commercial CFD code ANSYS Fluent 14.0 [10].This code solves the full time dependent Navier-Stokes equations with a finite volume scheme. The boundary conditions at the left and right side of the cylinder are dedicated nonreflecting boundary conditions. The pressure at this boundary condition is applied by means of User Defined Function (UDF) such that the outgoing wave is not reflected and an incoming wave can be imposed. For the calculation it is assumed that the two waves can be linearly superposed and that the speed of sound is known. From a sample plane inside the domain the wave leaving the domain can be calculated one time step later at the boundary. The wave leaving the domain is superposed with the wave entering the domain and the resulting pressure is applied at the boundary. The boundary condition is similar to the one presented by Liao and Wong [11].

Next to the pressure, the temperature has to be imposed at the boundaries during the half cycle in which the flow enters the domain. The temperature is calculated assuming adiabatic wave propagation. Due to this assumption the distance between the screen and the boundaries has to be large enough that the wave is adiabatic at the boundary. The minimum entrance length $L_{1}$ can be estimated as:

$$
L_{1}=\left(\delta_{\kappa}+2 \xi_{1}\right) S
$$

where $S=2$ is chosen as safety factor, $\delta_{\kappa}$ is the thermal penetration depth defined as:

$$
\delta_{\kappa}=\sqrt{\frac{2 \mu}{\omega \rho}}
$$

and $\xi_{1}$ is the particle displacement amplitude:

$$
\xi_{1}=\frac{\left|u_{1}\right|}{\omega}
$$

$\mu, \omega, \rho$ and $u_{1}$ denote the dynamic viscosity, the angular frequency, the density and the velocity amplitude respectively. Calculating the boundary condition values from the sample plane couples the choice of the time step size $\Delta t$ to the spatial discretization $\Delta x$, as the wave has to propagate at least a distance of one $\Delta x$ during one time step $\Delta t$. In numerical simulations the number of cells the wave propagates within one time step is given by the Courant-Friedrichs-Lewy (CFL) number [12]:

$$
C F L=c_{0} \frac{\Delta t}{\Delta x}
$$

where $c_{0}$ is the unperturbed speed of sound. The CFL number has to be larger than one, but not so large that the wave travels through a substantial part of the domain, as this would violate the assumption of plane wave propagation.

In general, the dimensions of the regenerator are small compared to the wave length, thus the time step will also have to be small compared to the period. This makes the simulations computationally expensive as several wave periods have to be simulated. The geometry is defined by the thermal penetration depth (equation 2) in order to achieve the best possible heat transfer. Thus, a larger penetration depth leads to a larger geometry and due to the larger element size at the boundary to quicker simulations. For this reason the mean pressure is chosen to be $p_{0}=1 \mathrm{~atm}$. This choice of pressure leads to a larger thermal penetration depth compared to a pressurized configuration. The frequency is set to $f=100 \mathrm{~Hz}$, as it is used in thermoacoustic refrigerators [13].

The dimensions of the reference model are taken such that the opening $(2 \cdot h$, see Figure 2$)$ is two thermal penetration depths large. Due to the high heat capacity of the metal wire compared to the working gas, the wire diameter is not defined from a thermal point of view, but rather a production point of view. For this reason the mesh was chosen from available screens with the required opening and the smallest wire diameter. The definition of the lengths is given in Figure 2 and their values are given in Table 1. All spatial dimensions in this paper are normalized to the diameter $D=0.22 \mathrm{~mm}$ and are denoted by the asterisk *. 


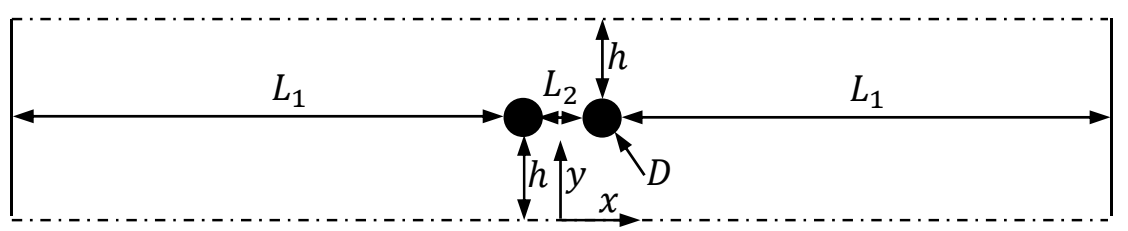

Fig. 2 Definition of the dimensions.

Table 1 Dimensions of the reference case normalized to the diameter $D=0.22 \mathrm{~mm}$.

\begin{tabular}{c|c}
\hline Length & Value \\
\hline$L_{1}^{*}$ & 39 \\
$L_{2}^{*}$ & 2 \\
$h^{*}$ & 3.5 \\
$D^{*}$ & 1 \\
\hline
\end{tabular}

The working gas used in the simulations is helium, as it is frequently used in thermoacoustic cryocoolers because of its low condensation temperature and its favorable heat transfer characteristics [14]. Helium is modeled according to the ideal gas law with the following properties at atmospheric pressure:

Table 2 Properties of helium at $p_{m}=1 \mathrm{~atm}$ and $T_{m}=300 \mathrm{~K}$.

\begin{tabular}{lccc}
\hline Description & Symbol & Value & Units \\
\hline Mean density & $\rho_{m}$ & 0.1626 & $\mathrm{~kg} / \mathrm{m}^{\wedge} 3$ \\
Dynamic viscosity & $\mu$ & $1.99 \cdot 10^{-5}$ & $\mathrm{~kg} /(\mathrm{m} \mathrm{s})$ \\
Thermal conductivity & $\lambda$ & 0.152 & $\mathrm{~W} /(\mathrm{m} \mathrm{K})$ \\
Specific heat ratio & $\gamma$ & 1.667 & 1 \\
Specific heat at constant pressure & $c_{p}$ & 5193 & $\mathrm{~J} /(\mathrm{kg} \mathrm{K})$ \\
\hline
\end{tabular}

All simulations are carried out for five wave periods, which represents a total simulation time of $t=0.05 \mathrm{~s}$. Looking at the heat flux at the most left point of the left cylinder (see Figure 3), shows that five periods is enough to reach a steady periodic state. The pressure amplitude of the incoming wave is set to $p_{1}=250 \mathrm{~Pa}$, which is low for a thermoacoustic application, but still allows the estimation of deviations from the thermoacoustic equations.

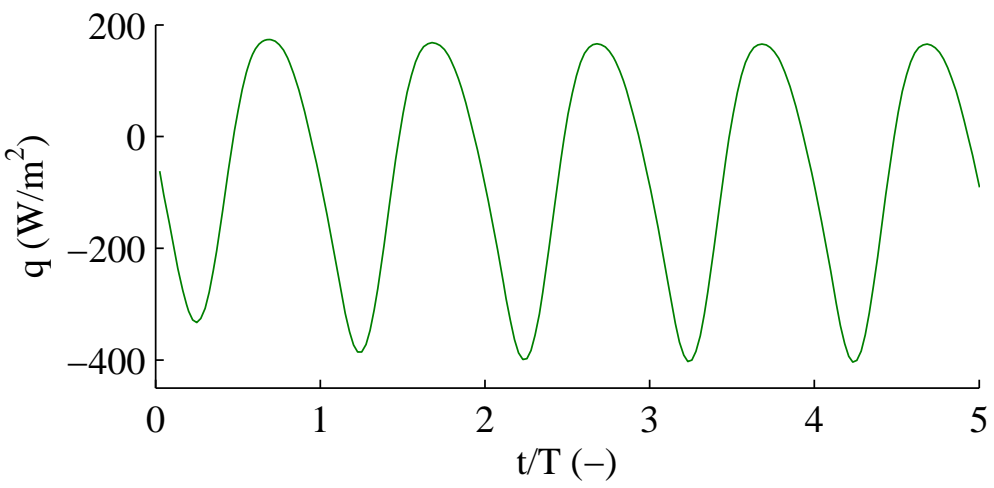

Fig. 3 Heat flux at the most left point of the cylinder, over dimensionless time. Heat flux going from the cylinder towards to fluid.

2.2 Numerical parameters In this chapter the numerical parameters for the above described model are given. Figure 4 shows the computational mesh. While the total domain height is shown, the domain extends further in the x-direction. An O-grid mesh is placed around each cylinder, with 120 grid points over the perimeter and 32 grid points in the radial direction, geometrically growing with a factor of 1.2 away from the cylinder. The rest of 
the mesh consists of rectangular elements with a constant height. At both sides of the cylinder the cells grow geometrically with a factor of 1.2 towards the boundary. This is possible as the gradients in the $x^{*}$-direction decrease with increasing distance to the cylinders. This is a consequence of the nearly adiabatic wave propagation occurring there. This has two positive effects: first, fewer elements are needed in the $x^{*}$-direction; secondly, due to the large element size $\Delta x$ at the boundary, the time step size can be increased. In the reference case this leads to a time step size of $\Delta t=1.5 \cdot 10^{-6} \mathrm{~s}$ which corresponds to more than 6600 time steps per period. The total number of elements is 23000 .

The simulations were conducted with the pressure based solver of Fluent 14.0 [10]. The pressure and the velocity were solved in a coupled manner. For the spatial discretizations the second order upwind scheme was applied and for the transient formulation the second order implicit scheme was used [15].

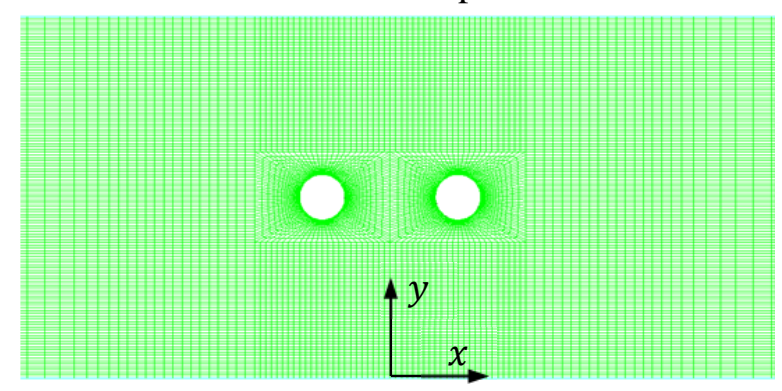

Fig. 4 Detail of reference mesh.

As a first step a mesh dependency study was carried out. The number of elements in each direction was doubled and the geometric growing factors set to 1.1. The results are compared with the reference mesh in terms of the heat transfer parameters presented in the next chapter. As a second step the entrance length is increased. In both cases the effects on the heat transfer parameters stayed below 1\%. It can thus be concluded that the mesh is sufficiently small to investigate the heat transfer characteristics of staggered screens.

\section{RESULTS}

The simulation results from the reference case are summarized in this section. The flow field and the heat pumping mechanism are presented. Finally, a parameter study is presented for which the height $h^{*}$ and the pressure amplitude $p_{1}$ are varied.

3.1 Flow field of reference case The time dependent and averaged flow field are presented in this section. The incoming wave travels through the domain and interacts with the two cylinders. Figure 5 (a) shows the velocity in the wave propagation direction over the $x^{*}$-coordinate. The velocity is normalized by the velocity amplitude of the boundary and is plotted at four different instances in time and at two different heights. The first height is on the symmetry boundary condition ( $y=0$, black solid line), the second goes through the center of the cylinder ( $y=h+D / 2$, red dashed). It can be seen that at all instances in time and for both heights the $x$-velocity is the same at the boundaries. Thus, the flow field is one-dimensional close to the boundaries.

Due to convection, a compression area develops in front of the cylinder and a wake area develops behind the cylinder. This is visible in the velocity profile at the instance in time $t=1 / 4 \cdot T$. The maximum velocity is not at $x^{*}=0$, but convected to higher $x^{*}$ values. Furthermore, the velocity profiles show a large asymmetry. In an acoustic code this would not be taken into account and the maximum velocity would be between the two cylinders at $x^{*}=0$. In Figure 5 (b) the normalized temperature oscillation over the dimensionless $x^{*}$-coordinate is shown at four different time instances of a period. It can be seen that heat is supplied to the left cylinder. At the instance in time $t=0 \cdot T$ the temperature for negative $x^{*}$-values is higher than the adiabatic temperature and will increase even further during the following quarter cycle due to compression. At the same time, heat will be convected to the regenerator material and the left cylinder will heat up. During the half cycle when the velocity is negative, the fluid temperature for positive $x^{*}$-values is lower than the cylinder temperature: due to the convection the right cylinder is cooled. Over a complete cycle the left cylinder will heat up and in the same manner the right cylinder is cooled. 

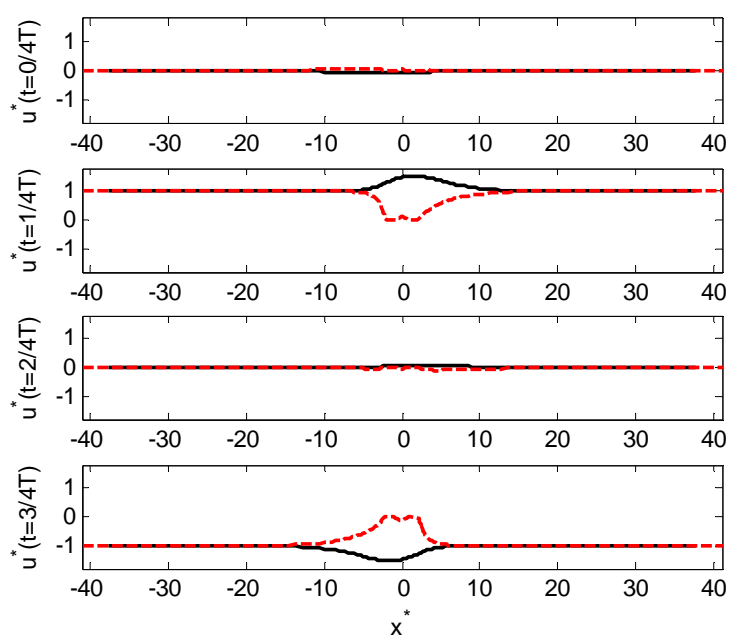

(a) $x$-velocity
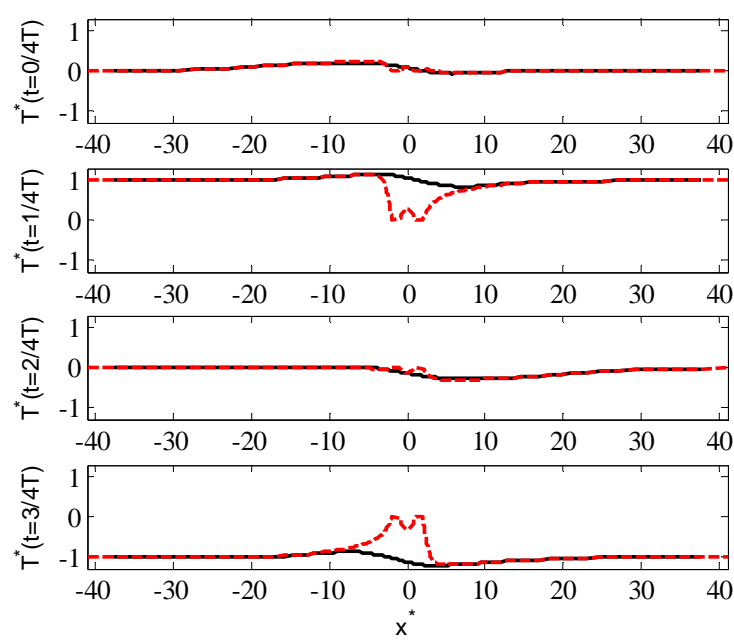

(b) Temperature oscillation

Fig. 5 (a) dimensionless velocity in wave propagation direction and (b) dimensionless temperature over $x^{*}$-coordinate at four different instances in time separated by a quarter period each and at two different heights (black line $y=0$ and red line $=h+D / 2$ ).

Due to convection, the gas undergoes a different thermodynamic cycle than the traveling wave Stirling cycle which would occur inside the regenerator. A p-v diagram can be drawn by following a gas parcel which starts at $x^{*}=0$ and $y=h / 2$ over several periods. Both the particle position and the p-v diagram are shown in Figure 6 (green line). It is a counterclockwise cycle, thus heat is pumped from the right cylinder to the left cylinder. The cycle that the gas parcel undergoes is shown schematically in Figure 7 and can be summarized by the following four steps:

(A-B) adiabatic compression

(B-C) isobaric cooling

(C-D) adiabatic expansion

(D-A) isobaric heating.

These four steps correspond to the ideal Brayton cycle [16].

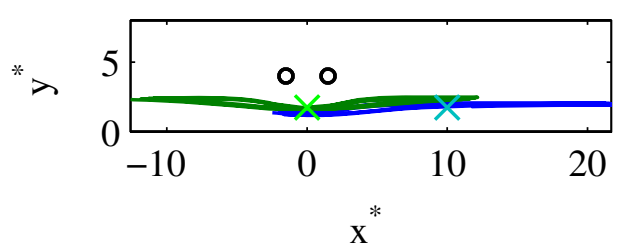

(a) Fluid parcel position

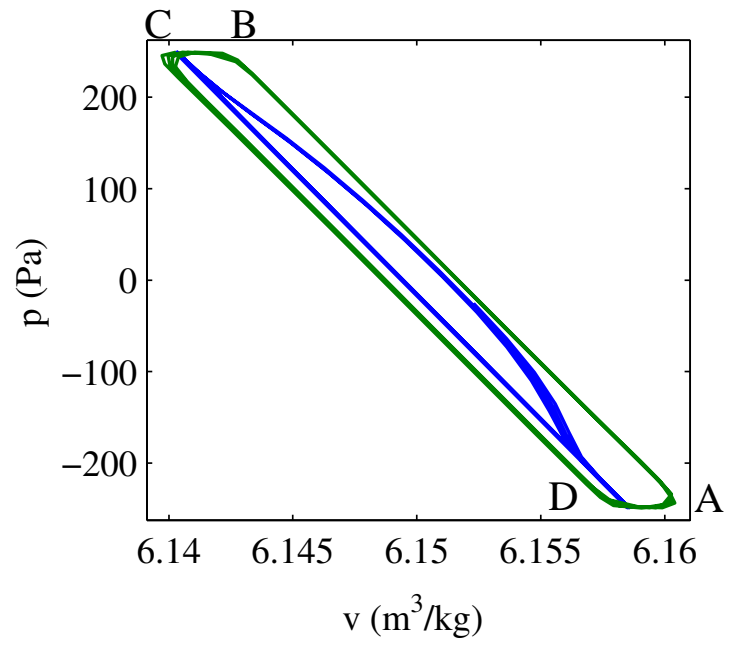

(b) p-v diagram of fluid parcel

Fig. 6 (a) position of the gas parcel over the last 4 periods, for two different starting positions (crosses): green $x^{*}=0, y=h / 2$ and blue $x^{*}=10, y=h / 2$; (b) p-v of the fluid parcel shown in (a). 


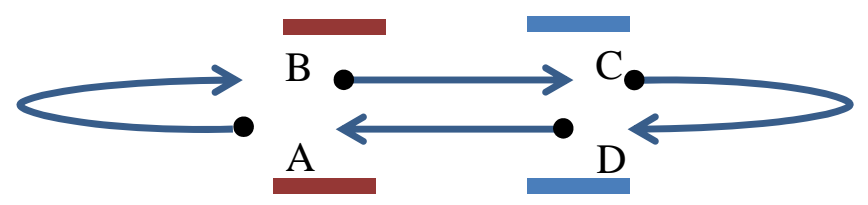

Fig. 7 Schematic of the thermodynamic cycle of the gas parcel: (A-B) adiabatic compression (B-C) isobaric cooling (C-D) adiabatic expansion (D-A) isobaric heating.

In typical cryocoolers operating at high amplitudes, the displacement amplitude is on the order of one tenth of the regenerator length [14], thus the cycle described above cannot be performed entirely. One adiabatic stage cannot be performed, for example stage (A-B) for a parcel entering the regenerator at the right. This stage will be an isothermal compression instead. However, heat pumping still occurs. To demonstrate this, a gas parcel is chosen that does not entirely pass the two cylinders. The starting position of this parcel is $x^{*}=10$ and $y=h / 2$. The position of the parcel and the p-v diagram are shown in Figure 6 (blue line). There are no isobaric stages (B-C, D-A), which means that the cooling and heating processes are no longer isobaric. Due to this, the enclosed area is smaller, but still heat is pumped at the extremity of the regenerator due to convective effects.

Another effect which is not resolved by the one-dimensional codes is the buildup of mean temperature around the cylinder, which is also due to the convective heat pumping described above. Gas which has a higher temperature than the adiabatic temperature amplitude is convected to the left cylinder, while gas having a temperature less than the adiabatic temperature amplitude is convected to the right cylinder. Figure 5 (b) illustrates this effect at time $t=1 / 4 \cdot T$ and time step $t=3 / 4 \cdot T$, respectively. Figure 8 shows the temperature oscillation averaged over one period, normalized with the temperature amplitude based on adiabatic wave propagation. The temperature is given for two different heights $y=0$ (black) and $y=h+D / 2$ (red) over the $x^{*}$-coordinate. The mean temperature reaches its maximum at the center $(y=h+D / 2)$ close to the cylinder. The temperature there corresponds to $20 \%$ of the temperature oscillation.

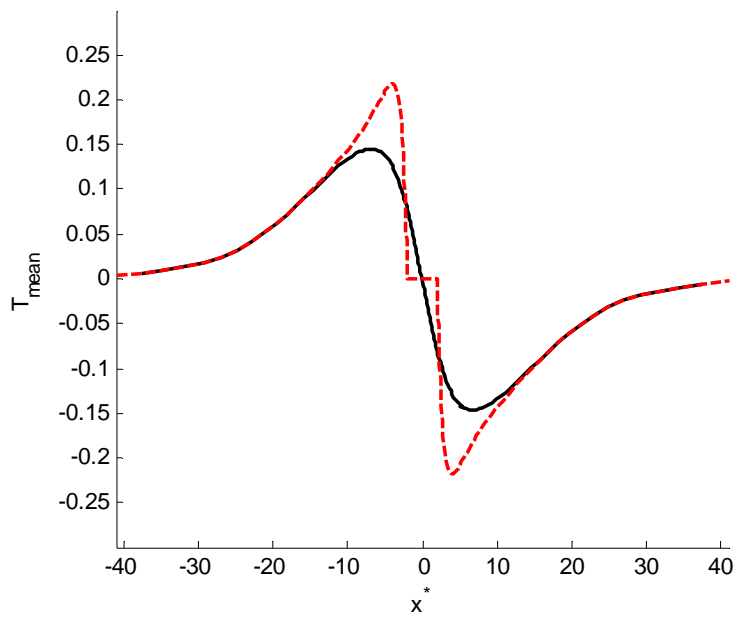

Fig. 8 Normalized temperature averaged over the last period as a function of the dimensionless $x^{*}$-coordinate for two different heights: $y=0$ (black) and $y=h+D / 2$ (red)

3.2 Heat pumping for different heights $\boldsymbol{h}$ In this subsection the heat pumped between the two cylinders, each representing one screen of the regenerator, is investigated for different heights $h$ and different pressure amplitudes $p_{1}$. To estimate the total heat transferred, the screen is assumed to be quadratic in shape with side lengths of $L_{y}=L_{z}=5 \mathrm{~cm}$. This means that the cylinders in our simulation represent wires with a length of $L_{z}$. Figure 9 (a) shows the heat transferred from the fluid to the right cylinder over the dimensionless half spacing $h^{*}$ for three different pressure amplitudes. It can be seen that for $h>\delta_{\kappa}$, no further heat can be transferred. With increasing pressure amplitude the heat transferred increases, but also the acoustic power dissipation increases as 
is shown in Figure 9 (b). Similar to the heat transfer, the dissipated acoustic power converges to a constant value. If the heat transfer and the dissipated acoustic power would be shown for the whole mesh and not for only one wire, they would go to zero as the number of cylinders per length $L_{y}$ decreases. Thus, from a power density point of view, a mesh with a maximal opening of twice the thermal penetration depth is optimal.

Given the heat transferred and the acoustic power dissipated, a coefficient of performance (COP) can be calculated as [16]:

$$
C O P=\frac{\dot{Q}_{c y l}}{P_{a c}}
$$

where $\dot{Q}_{c y l}$ is the heat transferred at the right cylinder and $P_{a c}$ is the dissipated acoustic power. The COP is shown in Figure 10 over the dimensionless half spacing $h^{*}$. As both cylinders have the same temperature which would result in an infinite COP in case of an ideal isentropic cycle, the COP's have to be compared with each other for the different configurations, instead of looking at the absolute values. While for low amplitudes a clear maximum can be seen for $h^{*} \approx 0.5$, this is not the case for higher amplitudes. One possible reason is that the displacement amplitude matches the length of the regenerator for an optimal cycle, as described above. Higher displacement amplitudes also lead to higher velocities around the cylinders, which shortens the cooling and heating phases and reduces the heat pumped during one cycle.

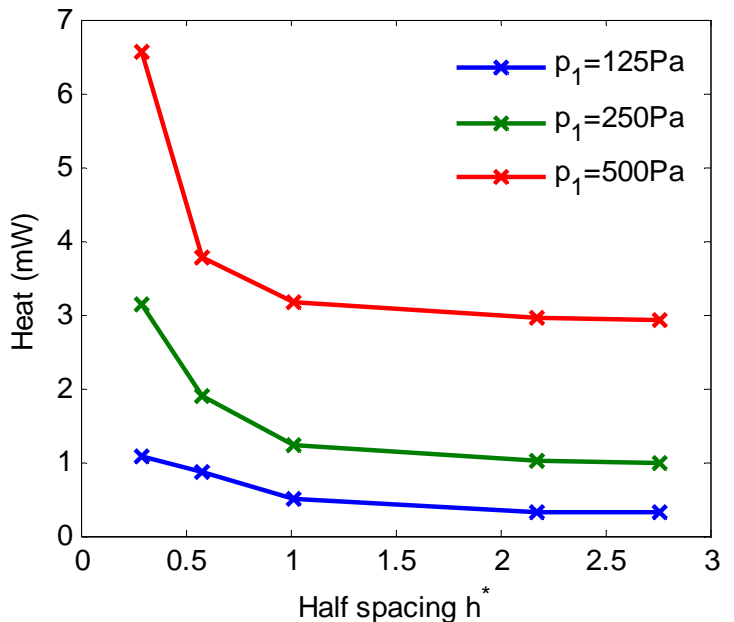

(a) Heat

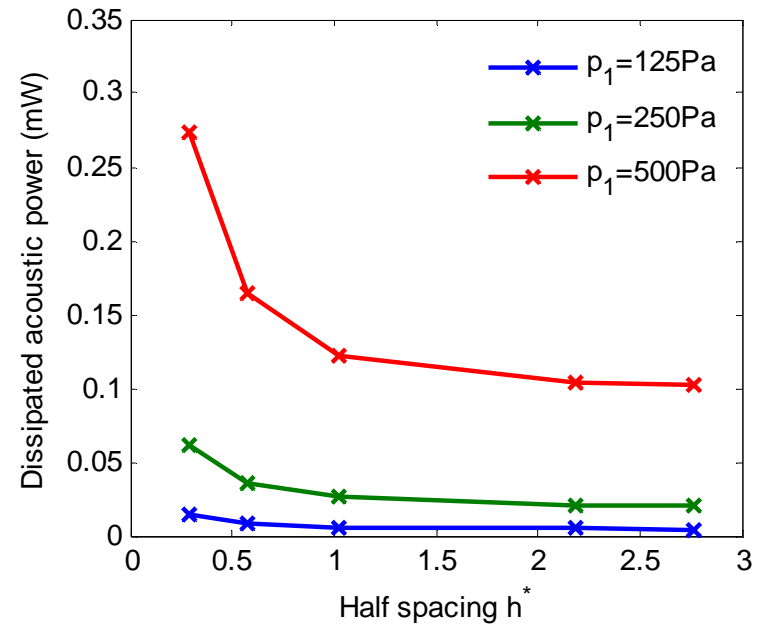

(b) Acoustic power dissipation

Fig. 9 (a) Heat transferred to one cylinder for three different pressure amplitudes over the dimensionless half spacing. (b) Dissipated acoustic power for three different pressure amplitudes over the dimensionless half spacing.

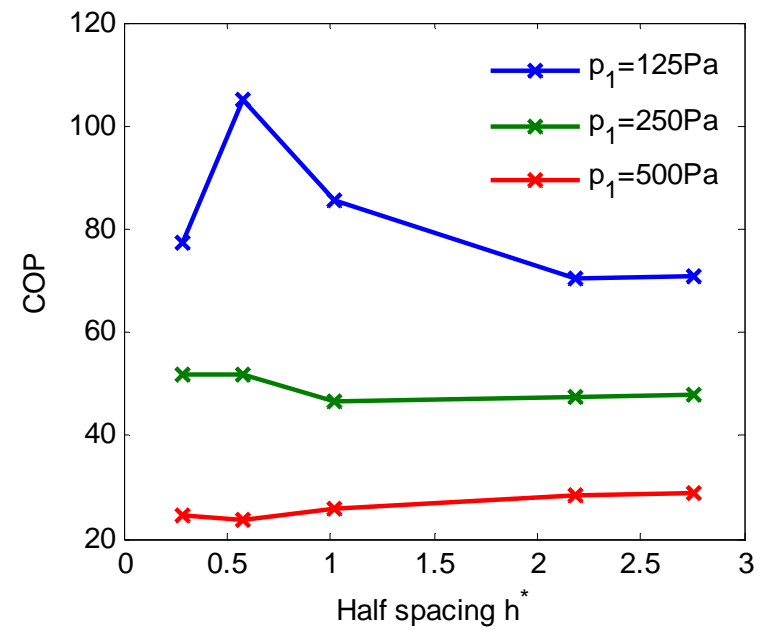

Fig. 10 COP for three different pressure amplitudes over the dimensionless half spacing. 


\section{CONCLUSION}

Convective effects play an important role in staggered screen regenerators; it is shown that the velocity profile shows convective effects as the position of the maximum velocity is shifted. Furthermore, the convection of heat leads to a thermodynamic cycle which can be approximated by a Brayton cycle. This cycle pumps heat from one stacked screen mesh to the other. The heat pumping was investigated for different half spacing $h^{*}$ and it was shown that from an opening corresponding to twice the thermal penetration depth on, no further heat is transferred. This has to be avoided if one hopes to achieve a high power density for a given cryocooler design. Moreover, the COP was given for three different pressure amplitudes over the dimensionless half spacing, but no common trend could be found.

In future work the trend of the COP will be investigated in more detail, as well as the effects of high amplitudes for which vortex generation occurs. A quantitative comparison between an acoustic code and the CFD can reveal further convective effects, even at low amplitudes.

\section{REFERENCES}

[1] Lewis, M., Kuriyama, T., Kuriyama, F., and Radebaugh, R., “Measurement of heat conduction through stacked screens,” , Advances in Cryogenic Engineering, 43, pp. 1611-1618, (1998).

[2] Ward, B., Clark, J., and Swift, G., Design Environment for Low-Amplitude ThermoAcoustic Energy Conversion (DeltaEC) Users Guide (Version 6.2), Los Alamos National Laboratory, (2008).

[3] Gedeon, D., (2013). Sage, Users Guide, Stirling, Pulse-Tube and Low-T Cooler Model Classes. 9th Edition.

[4] Williamson, C. H. K., “Sinusoidal flow relative to circular cylinders,” Journal of Fluid Mechanics, 155, pp. 141-174, (1985).

[5] An, H., Cheng, L., Zhao, M., and Dong, G., "Numerical simulation of the oscillatory flow around two cylinders in tandem,” Journal of Hydrodynamics, Ser. B, 18(3, Supplement), pp. 191 - 197, (2006), Proceedings of the Conference of Global Chinese Scholars on Hydrodynamics.

[6] Anagnostopoulos, P. and Dikarou, C., "Numerical simulation of viscous oscillatory flow past four cylinders in square arrangement," Journal of Fluids and Structures, 27(2), pp. 212 - 232, (2011).

[7] Mozurkewich, G., "Heat transfer from a cylinder in an acoustic standing wave,” The Journal of the Acoustical Society of America, 98(4), pp. 2209-2216, (1995).

[8] Gopinath, A. and Harder, D. R., “An experimental study of heat transfer from a cylinder in low-amplitude zero mean oscillatory flows,” International Journal of Heat and Mass Transfer, 43(4), pp. 505 - 520, (2000).

[9] Iwai, H., Mambo, T., Yamamoto, N., and Suzuki, K., "Laminar convective heat transfer from a circular cylinder exposed to a low frequency zero-mean velocity oscillating flow,” International Journal of Heat and Mass Transfer, 47(21), pp. 4659 - 4672, (2004).

[10] ANSYS Inc., “ANSYS FLUENT 14.0 User’s Guide”, (2013)

[11] Liao, Z. and Wong, H., “A transmitting boundary for the numerical simulation of elastic wave propagation,” International Journal of Soil Dynamics and Earthquake Engineering, 3(4), pp. 174 - 183, (1984).

[12] Poinsot, T. and Veynante, D., Theoretical and Numerical Combustion, 2nd Edition, R.T. Edwards, Inc., (2005).

[13] Radebaugh, R., “Cryocoolers: the state of the art and recent developments,” Journal of Physics: Condensed Matter, 21(16), pp. 164-219, (2009).

[14] Swift, G. W., Thermoacoustics: a unifying perspective for some engines and refrigerators, Acoustical Society of America through the American Institute of Physics, (2002).

[15] ANSYS Inc., “ANSYS FLUENT 14.0 Theory Guide”, (2013)

[16] Wu, C., Thermodynamics and Heat Powered Cycles: A Cognitive Engineering Approach, Nova Science Publishers,Inc., (2007). 